\title{
Enhances the Capacity of Load Adjusting by Improved Load Balancing Methodology in P2P Network
}

\author{
S. Govindaraj, Krishna Mohanta.S, C.Rukkumani
}

\begin{abstract}
To upgrades the limit of load modifying by working up a novel enhanced load adjusting philosophy in heterogeneous $P 2 P$ network with suitable load assignment and load reallocation process. We moreover propose another new adjusting procedure called two load adjusting strategy for peer to peer networks to make the best load designation assurance when a novel partner arrives. The new load adjusting method is also prepared to achieve the load reallocation immovably through framework running time, if congested peer arrives. In load altering estimation, no virtual servers are used. Subsequently, preparing overhead is diminished in light of restrictive meta-data preservation. Besides load adjusting additionally centers around controlling the framework action. The idea behind model is to investigate the impact of peer heterogeneity and to adjust the load dissemination in P2P frameworks
\end{abstract}

Keywords—load adjusting, peer to peer, TLBMP

\section{INTRODUCTION}

In the previous couple of years, peer-to-peer networks have turned into a well known strategy for broad content sharing. Not at all like ordinary client-server applications, P2P frameworks disperse the load of data storage, computation, communications and administration among a great many peers. Peers can join and withdraw the network whenever, at their will. Since the distribution of data can be random, the data stored in a P2P network is spread over countless. A standout amongst the most prevalent applications of P2P networks is file sharing. The current P2P frameworks are extensively arranged into two kinds: unstructured P2P networks and structured networks. For unstructured networks, the data objects don't have worldwide unique ids and queries are submitted as keywords. The peers in structured networks keep up unique distinguishing proof tag for each question. These days, the greater part of the peer-to-peer applications work on unstructured P2P networks. This architecture requests an exceptionally effective look system for the recovery of data [1]. A look for a question in a P2P network is fruitful on the off chance that it finds no less than one replica of the protest. Peers interface in an impromptu mold, the location of the reports isn't controlled by the framework and no guarantees for the achievement or the unpredictability of a hunt are offered [2]. Look strategies for unstructured networks can be assembled as either blind or educated. In a

Revised Manuscript Received on July 18, 2019.

S. Govindaraj, Research Scholar, Bharath Institute of Higher Education and Research.

Dr.Krishna Mohanta.S, Professor, Department of CSE, Kakatiya Institute of Technology and Science for women, Nizamabad, Telangana, India.

C.Rukkumani, Research Scholar, Bharath Institute of Higher Education and Research blind hunt, nodes don't store any data with respect to question locations. In educated methodologies, nodes locally store metadata that aides in the scan for the questioned objects. Existing blind strategies assault a ton of bandwidth to accomplish most extreme execution. Each hunt requires reaching a few nodes inside some separation called TimeTo-Live (TTL), making tremendous overhead to all nodes included. Educated strategies utilize their files to accomplish comparable quality outcomes, and to contract overhead. The restriction of most educated strategies is the upkeep cost of the files following peers join/leave the network or refresh the objects in the common envelope. Load adjusting is a primary issue to be illuminated for the productive task of P2P networks. A large portion of the inquiry techniques cause genuine load adjust issues. One of the significant issues is to distinguish overloaded nodes and reassign the approaching loads to others. This paper proposes a Distributed Search Technique (DST) to give synergistic load adjusting among peers. The algorithm is defined with the point of accomplishing great reaction time, high hit proportion, low network activity and versatile conduct. The primary commitments of the proposed look algorithm are: Q-learning based pursuit, two-way load adjusting, need for particular nodes, control peer idea, and the application of inquiry history points of interest. Like random walk a blind hunt technique [3], the proposed strategy does not choose walkers randomly. Power peers and common peers together join the inquiry procedure. With a specific end goal to accomplish the destinations, the proposed seek conspire keeps up a couple of tables. The tables are refreshed by indexed lists. Queries are circulated to both common peers and power peers all the while with the goal that the swarming impact on a couple of peers can be diminished. In a P2P network, a couple of nodes are high degree nodes and lion's share of the nodes have less number of neighbors. Because of this, high degree nodes are questioned oftentimes, accordingly the execution is decreased because of overwhelming inquiry load. Concurrent seeking among common peers and power peers alone can't adjust the load successfully. Henceforth in the proposed conspire, question is steered to a power peer in light of load data gathered by portable specialists from different power peers $[3,4]$

Peer-to-peer (P2P) frameworks are an ebb and flow research center in PC frameworks and networking. Such frameworks are alluring in their capability to tackle the immense appropriated computation and storage resources in 
today's networks, without requirement for mind boggling and delicate centralized control. A center issue in peer-topeer frameworks is the distribution of items to be stored or computations to be done to the nodes that make up the framework. A specific worldview for such allocation, known as the dispersed hash table (DHT), has turned into the standard way to deal with this issue in research on peerto-peer frameworks [18]. A conveyed hash table interface actualizes a hash work that maps any offered thing to a specific machine in the peer-to-peer network. For instance, In [16] utilizes Consistent Hashing to relegate items to nodes: items and nodes are pseudo-randomly hashed to a roundabout address space and a hub stores all items whose addresses fall between the hub's own particular address and the address of the hub going before it in the address space. DHTs vary from customary hash tables in two key routes: First, notwithstanding the addition and cancellation of items, DHTs must help the inclusion and erasure of pails: as machines join and leave the network, items must be relocated to different machines and the hash work updated to mirror their new location. Second, some sort of directing protocol is typically fundamental: since it isn't achievable in a P2P framework for each hub to keep up avant-garde learning of every other hub in the framework, a thing is gazed upward by following a succession of steering jumps through the peer-to-peer network. A substantial number of algorithms have been proposed [12] to give conveyed hash table usefulness. The dominant part of them require every hub of a n-hub P2P framework to monitor just "neighbor nodes" and permit the machine in charge of any key to be turned upward and reached in directing bounces. As of late a few variations have been proposed [14] that help queries with just consistent neighbor degree; this is hypothetically ideal yet might be unwanted practically speaking in light of adaptation to internal failure contemplations. An imperative issue in DHTs is load-adjust the even distribution of items to nodes in the DHT. All DHTs endeavor to load-adjust; for the most part by (I) randomizing the DHT address related with everything with an "adequate" hash capacity and (ii) making each DHT hub in charge of an adjusted segment of the DHT address space. Harmony is a prototypical case of this approach: its "random" hashing of nodes to a ring implies that every hub is in charge of just a little interim of ring address space, while the random mapping of items implies that exclusive a set number of items arrive in the ring interim possessed by any hub. This endeavor to load adjust can bomb in two different ways. To start with, the average random segment of the address space to nodes isn't totally adjusted. A few nodes wind up in charge of a bigger segment of the addresses and in this manner get a bigger part of the randomly appropriated items. Second, a few applications may block the randomization of data items addresses. For instance, to help go seeking in a database application the items may should be put in a particular request, or even at particular addresses, on the ring. In such cases, we may discover the items unevenly appropriated in address space, implying that adjusting the address space among nodes does not adjust the distribution of items among nodes. We offer protocols to tackle both of the load adjusting challenges simply depicted [5].

Be that as it may, such an immaculate world isn't sensible. All things considered, DHT based P2P frameworks still have serious load awkwardness issues [9]. Clearly, the peer who possesses a bigger zone needs to take more obligation than the peer with a littler one. Also, PC resources, for example, computational power, and storage limit and network bandwidth are very unique among peers. In[] led an estimation ponder, they found that the size of these resources could differ in the vicinity of three and five request of extent over the peers. On the off chance that a major zone is assigned to a frail peer, even with a uniform workload distribution, genuine load irregularity issue may happen. There are numerous different factors have the considerable effect on the framework execution too. For instance, the file ubiquity. Access frequencies of files may fluctuate in the three requests of extent. In [11] found that the most prominent $10 \%$ files represent just about $60 \%$ of overall network movement. The sizes of files are likewise very extraordinary. The length of the littlest file may contain many bytes just, while the greatest file could be a few GB long. Therefore, unique measures of PC resources are expected to serve diverse files. The locations of files may influence the framework execution also. Bringing a file from a remote peer devours substantially more bandwidth, and result in a long recovering dormancy. Consequently, the overlay network topology has its own belongings. Such a skewed file distribution should be all around considered in load adjusting algorithm plan. Be that as it may, all the current DHT load adjusting algorithms disregard no less than at least one factors and can't ensure the best execution. In this paper, we propose another load adjusting algorithm to tackle the above issues. In our answer, file get to history data is gathered and stored on each peer. This data together with peer heterogeneity are utilized for settling on load distribution and redistribution choices. The measure of the zone each peer possesses is dynamic; it can be changed during framework running period.

The remainder of this paper is organized as follows. Section 2 reviews the related work. An overview of the proposed search technique is given in section 3. Section 4 discusses the simulation methodology. Section 5 concludes the paper.

\section{RELATED WORKS}

While much research has been done on directing in P2P networks, work on effective load adjusting and complex queries in $\mathrm{P} 2 \mathrm{P}$ is just in its starting stages. Most structured $\mathrm{P} 2 \mathrm{P}$ frameworks essentially expect that items are consistently circulated on nodes. Two protocols that accomplish close ideal load-adjusting without the utilization of virtual nodes have as of late been given [7]. Our plan enhances them in three regards. In the first place, in those protocols the address relegated to a hub relies upon whatever is left of the network, i.e. the address isn't chosen from a rundown of conceivable addresses that exclusive rely upon the hub itself. This makes the protocols more powerless against noxious assaults. Second, in those 
protocols the address assignments rely upon the development history, making them harder to break down. Third, their load-adjusting guarantees are appeared for the "additions just" case, while we likewise handle cancellations of nodes and items. Work on load adjusting by moving items can be found in [6]. Their algorithm is like our own, anyway it just works when the arrangement of nodes and items are settled, and they give no provable execution guarantees, just test assessments.

Flooding based inquiry is widely utilized as a part of unstructured P2P networks like Gnutella. Flooding plans create a lot of network activity. To conquer this issue, a random walk [3], [4] method is frequently utilized. While this approach figures out how to lessen messages essentially, it demonstrates low execution on account of its random character and failure to conform to various inquiry loads. The proposed system makes utilization of Q-table data for choosing walkers. Versatile Probabilistic Search (APS) [5] advances a solitary file look into inquiry probabilistically in light of the question history and the conjectures of question sources. APS can be seen as an impromptu application of support learning [6]. APS appoints break even with status for every one of the nodes in the network while looking, without considering the nodes' degree, number of accessible objects and storage. Our strategy does not take after probabilistic sending; as an option, it utilizes Q-learning for choosing peers.

In Gnutella UDP Extension for Scalable Searches (GUESS) [], each ultrapeer is connected to different ultrapeers and to set of leaf-nodes. During a hunt activity, distinctive ultrapeers are iteratively reached taken after via seeking in their leaf-nodes. Be that as it may, the request in which ultrapeers are picked isn't indicated [8]. In [14], while a super-peer gets a question from a leaf hub, it advances it to suitable leaves and to its neighboring super-peers. In Intelligent-BFS [9], nodes keep up tables to store question neighborID tuples for as of late reacted demands from their neighbors. The precision of the algorithm relies upon the suspicion that nodes spend significant time in specific reports [19]. Fortification learning based pursuit [12] investigates new ways by sending queries to randomly picked neighbors. It chooses the best way from the returned comes about. A few arrangements have been proposed to address the load adjusting issues in structured P2P networks utilizing the idea of virtual server [6]. None of the procedures is suitable for unstructured P2P networks. The unstructured networks do not have the conveyed hash tables and unique identifiers. Due to these, load adjusting is considerably harder in unstructured networks. Our plan uses a disseminated load adjusting plan for enhancing look execution in unstructured networks.

A hypothetical examination of a comparable protocol was given in [12]. In their setting, nonetheless, items are thought to be employments that are executed at a settled rate, i.e. items vanish from nodes at a settled rate. Besides, they investigate the average sit tight time for employments, while accomplish load adjust. Complex queries, for example, extend looks are likewise a developing research topic for $\mathrm{P} 2 \mathrm{P}$ frameworks $[5,6]$. A proficient range seek data structure was as of late given in [3]. In any case, that work we are more inspired by the total number of items moved to

does not address the issue of load adjusting the quantity of items per hub, making the streamlining supposition that every hub stores just a single thing. In ongoing free work, in [4] consider a load adjusting plan like our own and call attention to applications to go seeks. Be that as it may, their plan depends on having the capacity to rapidly locate the slightest and most loaded nodes in the framework. It isn't clear how to help this activity effectively, and without making overwhelming network movement for these nodes with extraordinary load

\section{TWO LAYER LOAD BALANCING METHODOLOGY FOR PEER TO PEER NETWORKS (TLBMP)}

\subsection{Two-Layer Model}

In this area, we depict another framework for adjusting load in P2P. In spite of the fact that it has been outlined with an emphasis on the frameworks with time-expending question handling, it is pertinent on any P2P. An overload of a hub might be caused by an extensive data volume stored at the hub. One of the activities suitable in such a circumstance is moving piece of the data to a less loaded neighboring hub. Tragically, this task isn't accessible in all structures since the data are not really totally arranged and extend parceled. A general arrangement of this issue is supporting presence of a few "consistent nodes" at one physical host. This gives an alternative of part the overloaded hub and moving another hub to a less loaded host. Another inspiration for utilizing this model is supporting frameworks with various overlays architecture, for which a few nodes for each host is an essential prerequisite. The depictions underneath abridge the documentation utilized as a part of the accompanying content. Nodes are the consistent units of the structure being adjusted and shape the intelligent layer. Every hub comprises of the storage and the steering structure (connections to different nodes and the route system). Hosts allude to the physical PCs. The sensible nodes are mapped to the arrangement of physical hosts [14].

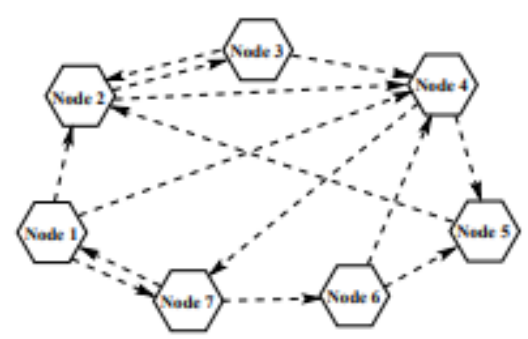

Fig.1 The logical layer

On a fundamental level, a peer is made by acknowledgment of a hub at a physical host. The two-layer architecture is outlined out in Figure 1. The load-adjusting activities may alter the coherent layer, while the arrangement of accessible hosts is overseen from the outside of the framework. The use of the adjusting framework is completely straightforward for the outside condition all 


\section{ENHANCES THE CAPACITY OF LOAD ADJUSTING BY IMPROVED LOAD BALANCING METHODOLOGY IN P2P NETWORK}

tasks of the specific P2P, for example, Insert or Search can be postured to the framework. By and large, when a hub gets an inquiry ask for, it can either forward it to different nodes or assess it on its nearby data or both. In the framework, the sending has dependably need over every single nearby datum look demands at specific host. The load-adjusting component regards the arrangement of hosts as settled and endeavors the accompanying adjusting activities that influence the legitimate layer of the framework. The Split task parts the data of a hub similarly and places the new hub at another host. This task is bolstered by all P2P known. The reciprocal activity, Leave, is either executed as a converge of the hub with some other hub or the data are re-embedded to the structure after a hub erasure. The load-adjusting may require relocating a hub to another ordinarily less-loaded host. In the event that the relocated hub knows all nodes that point to it, at that point this activity can be executed by informing every one of these nodes about the host change. Something else, the Migrate task is acknowledged as a couple of sequential activities leave and join standard gave by all P2P. The framework abuses replication as one of the load-adjusting instruments. Task Replicate makes a replica of the predefined hub at another host. Once a hub is replicated, it can choose to forward a hunt question to a chose replica as opposed to preparing the inquiry locally. Choices about inquiry sending are constantly made at the fundamental hub the replicas don't have any directing data. The Unify activity evacuates a particular replica of given hub. The compositions of the load-adjusting activities are delineated in Figure 2.

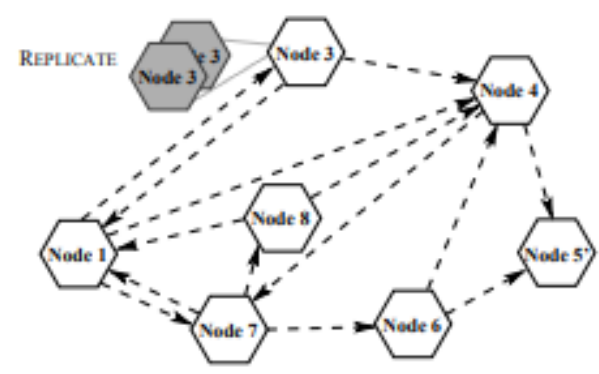

Fig. 2. Replicate operations.

\subsection{Measuring the Load}

To start with, we make a network model to depict the load adjusting issue in DHT-based P2P frameworks. Expect there are $\mathrm{N}$ peers in a DHT framework, their abilities are $\mathrm{C} 1, \mathrm{C} 2$, ..., $\mathrm{Cn}$, the zones possessed by these peers and the workloads on these peers separately. The framework contains files circulated on these peers, they have the diverse lengths. In this way, they have distinctive access overhead. For these files, we accept the comparing access overheads are and the entrance frequencies [17]. Under the perfect circumstance, framework workload ought to be very much conveyed on the peers corresponding to their abilities. We gauge the workload on a peer $\mathrm{K}$ as the aggregate of access frequencies for every one of the files whose fields are fallen into in the previous history data recording period. Expect those files are a1, a2, ... ae. To accomplish the ideal load adjusting, the proportion of the workload on an offered peer to the overall framework workload ought to be relative to the proportion of its ability to the amassed limit everything being equal. In such case, the workload is very much appropriated on every one of the peers as indicated by their abilities. Notwithstanding, as a general rule, such a perfect workload distribution is difficult to create because of the accompanying reasons:

1) we can't get the precise file get to recurrence data;

2) A DHT based P2P framework is completely conveyed, no peer can have a worldwide perspective of the entire framework, and no peer knows every other peer and additionally their workloads. It is difficult to compute the overall workload distribution by utilizing the above equation. To keep the framework in the ideal adjusted workload distribution will acquire critical overhead;

3) A DHT based P2P framework is dynamic, the file get to practices change every now and then.

An immaculate workload distribution at a specific time may not be the best arrangement at some other time. Then again, a definitive motivation behind load adjusting is to accomplish the most reduced average client saw file get to idleness. It isn't important to have the ideal load distribution. As per the lining hypothesis, on the off chance that a server isn't overloaded, the average reaction time increments gradually as we include more errands it. Be that as it may, if the server is overloaded, the average reaction time will increment definitely just on the off chance that we continue including more errands. From the above exchange, we presume that in DHT frameworks, it isn't conceivable and not basic to accomplish the ideal load adjusting in the worldwide level. Our answer intends to give the best load adjusting property for little districts. We accept on the off chance that we can ensure the ideal workload distribution for every little area, we can safeguard relative great load adjusting property for the entire framework. In our load adjusting system, we utilize the history table to gauge the future file get to recurrence. The algorithm contains two stages: First, when another peer comes, we endeavor to accomplish the best static workload distribution task as we can; Second, during the framework running time, we play out the workload modification activity just when it is important.

\subsection{Balancing Strategies}

So as to make it suitable to fill in as the overlay administration framework for our load adjusting approach. The load-adjusting algorithm has additionally been altered to exploit the new overlay structure. We expanded the fundamental TLBMP protocol with decides that help the clustering of same-type peers; on top of that productive topology, self-composed load-adjusting is connected to the lined solicitations for the different service writes dwelling in a decentralized service network. TLBMP goes for choosing the most astounding limit peers to fill in as super peers. The principal application-particular outline choice we made for our load-adjusting situation was in this way to delineate limit of a peer to the measure of service asks for that peer can satisfy in a time unit. This, thus, is reflected in the

Blue Eyes Intelligence Engineering

\& Sciences Publication

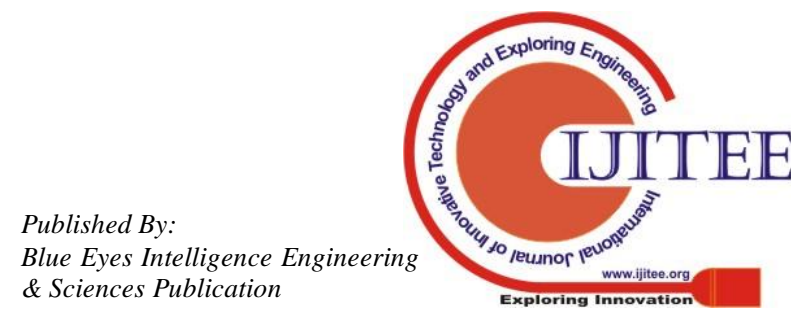


TLBMP overlay as the objective number BS of client peers kept up by a super peer, as we need higher limit peers to interface with an extensive number of lower-limit same-type neighbors. Along these lines, super peers are very much situated to effectively adjust their neighbors' demand lines. Besides, as TLBMP had initially been produced to oversee peers regardless of service compose, another augmentation to the protocol was to make it mindful of peer writes. A peer may have at any given time both same-type and diverse write neighbors, however, as talked about in segment II, a peer can just perform load-adjusting activities with sametype peers. In this manner diverse sorts of principles apply when that peer deals with its connects to same-type versus diverse write neighbors, to guarantee the incremental development of neighborhoods in the overlay, in which at least one 1 peers total and serve various biomass peers of a similar kind. The present execution accept that peers give just a single service write, and henceforth take an interest in just a single group. Under this model, peers could offer in excess of one service write by running different occasions of the protocol. Here we display comes about for single-type peers just; extending to enable peer to offer numerous service composes is arranged as future work [19].

To empower the clustering of same-type peers utilizes an arrangement of guidelines that manage the collaborations of same-type versus distinctive compose peers. We center here around those standards, and for the most part overlook the mechanics of super peer determination, advancement and downgrade, which continues as before as in the TLBMP fundamental protocol. Biomass Peers: All peers start protocol execution as biomass. A separated biomass peer $b$ will endeavor to associate with an extending super peer it can discover through the lower-level babble protocol. Notwithstanding, it will just choose one of its same kinds. On the off chance that no suitable extending super peer can be found, $b$ will elevate itself to extending status. This decide guarantees that there are continually extending e of each kind in the network, since these self-chose extending peers must go about as total focuses for disengaged peers of their write. On the off chance that a biomass peer ever winds up detached, it looks for another extending to interface with, as during bootstrapping. Peers - all states: L peers total biomass peers of their same sort up to an objective level BS to that end; peers in a higher protocol state will dependably attempt to "pull" biomass from other neighboring peers of their write in bring down states, or in a similar state however of lower limit, through the execution of assimilation rules. peers additionally frame the foundation of the overlay, and are in charge of its strength; to that end, peers endeavor to make and keep up an objective number of connections CS to other e of their write, which is a parameter of the protocol. In there is another parameter, as peers likewise attempt to keep up $\mathrm{CO}$ connects to e of various kinds. CO guarantees that groups don't wind up detached from each other. Expanding the CS or CO parameters builds the quantity of cross-associations, while adding overhead to the protocol. CO guarantees that bunches don't end up separated from each other. L peers go about as sort go betweens for different peers; if a $\mathrm{h}$ has a neighbor $\mathrm{n}$ of an alternate kind, $\mathrm{h}$ will check if some other of its neighbors $s$ is of an indistinguishable sort from $\mathrm{n}$. Assuming this is the case, $\mathrm{h}$

will exchange $\mathrm{n}$ to wind up a neighbor of s. If not, $\mathrm{h}$ attempts a similar thing with the neighbors of the majority of its own neighbors. h won't really drop its connect to $\mathrm{n}$, on the off chance that it stays inside the farthest point of its objective CO distinctive compose $\mathrm{L}$ joins. $\mathrm{L}$ peers additionally execute rules particular to their protocol state, as portrayed beneath. Extending Peers: Super peers stay in the extending state until the point that they have achieved their objective number of biomass neighbors of a similar kind BS, and soon thereafter they elevate to spreading status. Extending peers likewise stay themselves to the overlay with a solitary connect to either a fanning or a stable peer of any sort. In the occasion two extending peers of a similar sort move toward becoming neighbors, the lowerlimit peer will exchange the greater part of its biomass peers to the higher-limit and downgrade itself to biomass status. This is an assimilation run the show. Fanning Peers: One essential capacity of these super peers is to develop new connects to other super peers, in this manner building strong cross-associations for the overlay. Fanning peers are those that have amassed their objective number of same-type biomass peer (BS), however have not yet achieved their objectives of CS and CO connects to other super peers of a similar kind and distinctive writes, individually. Concerning same-type joins, if a stretching peer hb is under the objective number CS, it will look through its neighborhood to decide whether any of its neighbors are appended to a suitable same-type L peer that isn't now its own particular neighbor. Regarding diverse compose $\mathrm{L}$ joins, if $\mathrm{hb}$ is under its objective $\mathrm{CO}$, it will randomly choose a peer from the babble store and grow a neighbor connect to it. The subsequent peer may be of any kind (counting the same). A fanning peer likewise endeavors to dependably make them expand peer among its neighbors; if no such peer exists, it will pick its biggest limit biomass tyke and advance it, acquiring an extending peer of its same sort. On the off chance that a stretching peer ever gets over its biomass limit, it will drive overabundance biomass kids to a connected same-type super peer. When it has developed CS and CO interfaces, an expanding peer will elevate to stationary state. Fixed Peers: Once a peer has accomplished fixed status, it will endeavor to keep up it through ingestion, that is, by pulling biomass from bring down condition of its same sort. Also, it will attempt to develop new connections if some are lost, with a specific end goal to keep up the objective CS and $\mathrm{CO}$. On the off chance that the stable peer, rather, happens to have more than CS connects to same-type super peers, or more than $\mathrm{CO}$ connects to various sort super peers, it will randomly drop the abundance joins. A stationary peer ought to be associated with either zero or one same-type extending peers. In the event that it has numerous extending neighbors, it will associate them together, in this way triggering the ingestion run the show. On the off chance that it has both fanning and extending neighbors, it will exchange the extending peers to wind up offspring of the stretching peers. With a specific end goal to keep the overlay from sinking into nearby ideal, stable peers will

Published By: 

IN P2P NETWORK

sporadically grow another random connection by picking a random from the prattle reserve and associating with it. The expansion of the principles depicted above to the essential TLBMP protocol empower to rapidly sink into an overlay design where peers of each sort are thought around, and associated with, at least one peer of that write.

Load-adjusting is - as before - performed between neighbors. Nonetheless, in the past work, since all peers were viewed as homogeneous in their figuring capacities, the objective of the algorithm was to adjust the lines of service demands for neighboring peers as consistently as could reasonably be expected. In TLBMP, as a result of the heterogeneous limit of peers, the objective of load-adjusting progresses toward becoming making line lengths corresponding to the limit of each peer. In view of this standard, when a load-adjusting activity is played out, a peer dad chooses a random same-type neighbor $\mathrm{pb}$. On the off chance that dad is a biomass peer, it will have just a solitary neighbor, a same-type. In the event that dad is a L peer, it will just attempt to adjust its line with other same-type $\mathrm{L}$ peers; as biomass peers have just a solitary neighbor, they will be the ones to start the load-adjusting activities with their parent. Along these lines, load-adjusting tasks have a tendency to happen specially between the more fit super peers, which helps ensuring that employments will be adjusted all through the group. dad and pb analyze their line lengths; Perfect line lengths are figured by deciding the total number of employments in the two lines and isolating the occupations relatively in light of dad and pb's abilities. On the off chance that the lines are lopsided, occupations are exchanged from the line that is over its optimal length to the line that is under that length. During load-adjusting tasks, the exchange of a vocation includes just an exchange of a reference to the activity, that is, to the location where that activity can be really recovered when a peer is prepared to execute it. The decision of sending just references keeps the network overhead as little as could reasonably be expected, since occupations could be made out of a lot of data.

\section{EXPERIMENTAL EVALUATION}

Table 6.1 Parameter settings

\begin{tabular}{|l|l|}
\hline Parameters & Values \\
\hline Simulator & Ns2 \\
\hline Data Set & UCI \\
\hline No of nodes & $25-250$ \\
\hline Load size & $20 \mathrm{k}-100 \mathrm{k}$ \\
\hline
\end{tabular}

Test on load adjusting algorithm is led and assessed with before arrangements. In the first place, the reenactment condition is clarified in analyses, and after that the outcome is created. In reenactment, the burglaries of intra-travel field joins, stub-travel relations and intra-stub area relations are situated to 100, 20 and 5ms separately. The quantity of files in the plan is varied from $20 \mathrm{~K}$ to $100 \mathrm{k}$ two-way load adjusting technique for peer to peer networks (TLBMP) is productively composed and actualized in NS-2 simulator which is appeared in table 6.1. Likewise reproductions are done to demonstrate the adequacy of the TLBMP work with seat stamp data sets got from UCI Repository. With the load request factor acquired from the peer nodes preparing capacities, peer servers handling cycle necessities are distinguished. Load redirections are made to peer server nodes at whatever point the peer hub gets overloaded. The differing data nature of the charge required by the peer servers is totally perceived. Data Format Load Points are composed to display data configuration involving specific nodes and its motivation on load adjusting the peer servers are considered. The execution of the upgraded load adjusting system in heterogeneous P2P networks is estimated regarding Load development factor, Energy utilization and Average overall execution time. The test is to accomplish the most ideal tradeoffs between load development factor and hub usage clashing measurements.

\section{RESULTS AND DISCUSSION}

The proposed upgraded load adjusting procedure in heterogeneous P2P networks is distinguished to be better contrasted with a current work, for example, HPSC for productively dealing with the load in network [] and proposed Enhanced Load Balancing Strategy in Heterogeneous P2P Networks (ELBSHN) and Node determination methodology by breaking down through the test assessment. The underneath table and chart depicts the execution of the proposed TLBMP.

\subsection{Energy Consumption}

Vitality utilization is characterized as measure of vitality expended in improved load adjusting plan for heterogeneous P2P frameworks. Vitality spared during the hub correspondence chooses the capability of the framework. More vitality utilization prompts better framework execution. Vitality utilization is estimated as far as rate (joules). The table beneath depicts the execution of existing ELBSHN, HPSC and proposed TLMBP. Table 5.1 depicts the utilization of vitality to share their data in a specific interim of time with the nodes in the P2P network. The aftereffect of the proposed Enhanced Load Balancing in Heterogeneous P2P Network is contrasted and Heterogeneous Peer Node Server Cycle Analyzer utilizing DCDA system and Node Selection Strategy and existing ELBSHN. Figure 6.3 portrays the utilization of vitality charged when number of nodes in the network increments to process the suitable data. TLMBP expends less vitality for around 13-16 \% to play out a load adjusting process contrasted with ELBSHN and HPSC. Load allocation process in TLMBP legitimately balances the hub among the peer nodes with no flood. Also load reallocation process handles blockage just when hub congested so vitality utilization is high in TLMBP. The landing of an asset assert protest at a coordination service in ELBSHN strategy lines it in the claim list using more vitality. 


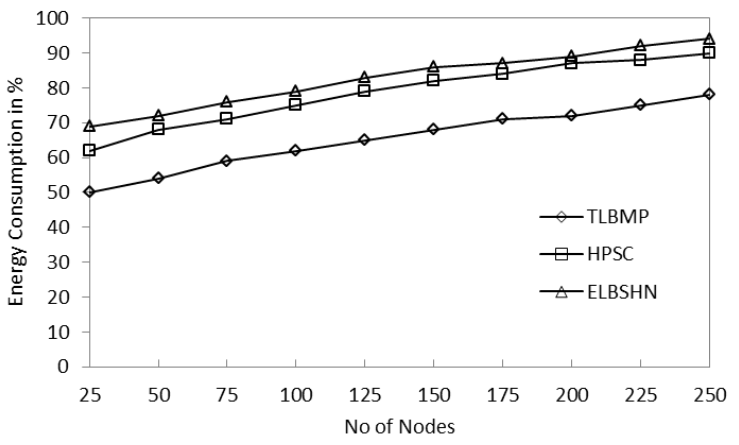

Figure 3. Energy Consumption

\subsection{Node Utilization}

Hub usage is named as the best in overabundance of every virtual time $t$ of the 99 percentile of the uses of the nodes at occasion. In view of the load development factor the hub usage is chosen. Load development factor additionally called as the total affiliation cost is utilized to decide the total cost of influencing objects. Each point on the lower line conveys to the enhancements of our algorithm with a trustworthy choice of load adjust time T. The natural inclination is that as $\mathrm{T}$ diminishes, 99.9th percentile hub use diminishes however load development factor increments. One needs to give of choosing $\mathrm{T}$ to coordinate among these two measurements in the technique which is most suitable for the goal application. The current DCDA method must move each question once to place initially in network. A load development factor of 0.1 requires balancer which expends $10 \%$ bandwidth that is fundamental to interleave the items in the first place. The use of hub for load adjusting factor is resolved in view of the hub development factor in the dynamic P2P frameworks as delineated in Figure 6.4 catches the tradeoff between load development and 99.9th percentile hub usage. TLMBP gives better hub use of around 10-15\% contrasted with existing ELBSHN and HPSC as the workload inside these locales is reallocated dependably with peers abilities and the prospect file get to tendencies, bringing about better hub use. While utilizing ELBSHN, the helper methodology is summoned and additionally coordinated to accumulate the rundown of asset which covers with the submitted asset and thus the hub usage is influenced.

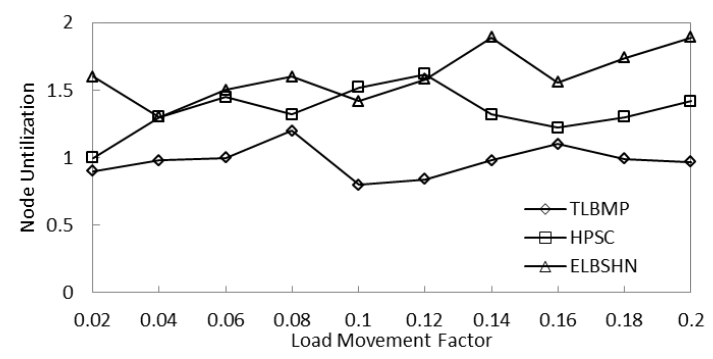

Figure 4. Node utilization

\subsection{Average Overall Execution Time}

Average overall execution time is characterized as the measure of time taken to play out the execution procedure. The procedure of load allocation in adjusting the load in view of zone division and load reallocation to understand the hub clog decides the average overall execution time. The overall execution process is estimated regarding seconds (sec). The aftereffect of the proposed Enhanced load adjusting in heterogeneous P2P network is contrasted and heterogeneous peer hub server cycle analyzer utilizing DCDA system and Node determination technique and existing ELBSHN. In light of the Table 5.3 a chart is appeared in Figure 6.5 outlines the average overall execution time required to play out the load adjusting strategies. TLMBP devours less time of around $20-25 \%$ to execute the load adjusting forms contrasted with ELBSHN and HPSC. The relative and precursor estimation in each round of the reallocation procedure lessens the initiated overhead and furthermore the execution time is limited all the while in the TLMBP. ELBSHN based hashing technique is used for hash procedure to control the focuses with the goal that the execution time taken is high in performing mapping through hash work

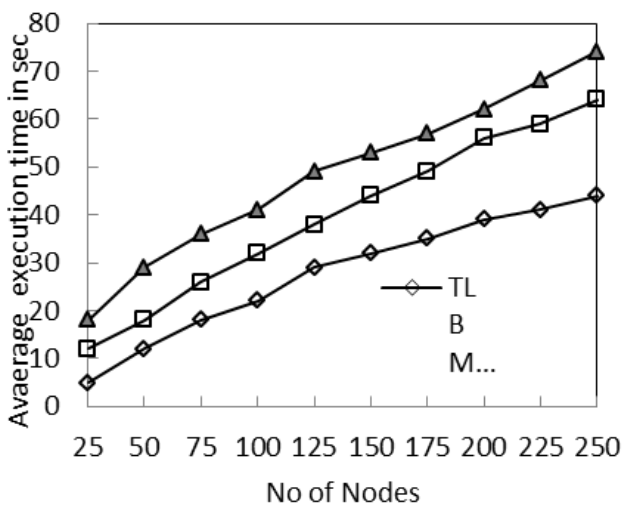

Figure 5. Average Execution time

\section{CONCLUSION}

Improved load adjusting procedure for heterogeneous $\mathrm{P} 2 \mathrm{P}$ framework is given better potential in adjusting the load utilizing load allocation and load reallocation. The load adjusting procedure analyzes the worry deliberately and frames the load adjusting game plan. Existing framework is incredibly misrepresented by framework properties, for example the peer heterogeneity in P2P overlie network topology. The upgraded load adjusting procedure considers every one of these worries to deliver better load adjust decisions. At first, TLMBP create an effective procedure to keep up the file get to record data and utilize the recorded data to anticipate the future file get to activities and furthermore with utilized data the way toward partitioning the workload is made more precise and produces upgraded load redesign decisions by receiving the sizes of nodes limits. Load adjusting algorithm, the load reallocation is accomplished when required and decreases the essential overhead. With TLMBP approach, load is well equally conveyed between various peers, the client affirmed recapturing overhead is dense and the bandwidth usage is lessened also. Reproduction comes about demonstrated that the proposed TLMBP conspire enhanced vitality utilization, framework limit as far as average overall execution time and asset usage. 


\section{REFERENCES}

1. Li. X, and Wu. J, "Improve Searching by Reinforcement Learning in Unstructured P2Ps," in 2004 Proc.26th Conf.Distributed Computing Systems ,pp. 75

2. Ananth Rao, Karthik Lakshminarayanan, Sonesh Surana, Richard Karp, and Ion Stoica, "Load Balancing in Structured P2P Systems," in Proc. IPTPS, Feb. 2003.

3. Suresh, K.C., Prakash, S, Priya, AE \& Kathirvel, A 2015, 'Primary path reservation using enhanced slot assignment in TDMA for session admission', The Scientific World Journal, Article: ID 405974.

4. John Byers, Jeffrey Considine, and Michael Mitzenmacher, "Simple Load Balancing for Distributed Hash Tables," in Proc. IPTPS, Feb. 2003.

5. Frank Dabek, Frans Kaashoek, David Karger, Robert Morris, and Ion Stoica, "Wide-area Cooperative Storage with CFS," in Proc. ACM SOSP, Banff, Canada, 2001.

6. David Karger, Eric Lehman, Tom Leighton, Matthew Levine, Daniel Lewin, and Rina Panigrahy, "Consistent Hashing and Random Trees: Distributed Caching Protocols for Relieving Hot Spots on the World Wide Web," in Proc. ACM STOC, May 1997.

7. M. Adler, Eran Halperin, R. M. Karp, and V. Vazirani, “A stochastic process on the hypercube with applications to peerto-peer networks," in Proc. STOC, 2003.

8. Palani1 U., Amuthavalli G., Chethan Prakash V. and Suresh K.C. "Energy-based Localization of IWSN in Biotechnology Industrial Applications” Res. J. Biotech ,Vol. (Special Issue II) August (2017)

9. Aberer, K., Datta, A., Hauswirth, M.: The Quest for Balancing Peer Load in Structured Peer-to-Peer Systems. Technical report, EPFL, Swiss (2003)

10. Ganesan, P., Bawa, M., Garcia-Molina, H.: Online balancing of range-partitioned data with applications to peer-to-peer systems. Technical report, Stanford U. (2004)

11. Crainiceanu, A., Linga, P., Machanavajjhala, A., Gehrke, J., Shanmugasundaram, J.: P-Ring: An index structure for peerto-peer systems. Technical Report TR2004- 1946, Cornell University, NY (2004)

12. Aspnes, J., Kirsch, J., Krishnamurthy, A.: Load balancing and locality in rangequeriable data structures. In: PODC '04: Proceedings of the twenty-third annual ACM symposium on Principles of distributed computing, New York, NY, USA, ACM Press (2004) 115-124

13. Karger, D.R., Ruhl, M.: Simple efficient load balancing algorithms for peer-topeer systems. In: SPAA '04: Proceedings of the sixteenth annual ACM symposium on Parallelism in algorithms and architectures, New York, NY, USA, ACM Press (2004) 36-43

14. Theoni Pitoura, Nikos Ntarmos, P.T.: Replication, load balancing and efficient range query processing in DHTs. In: Proceedings of the International Conference on Extending Database Technology (EDBT), Munich, Germany. (2006)

15. Navimipour, N. J., \& Milani, F. S. (2015). A comprehensive study of the resource discovery techniques in Peer-to-Peer networks. Peer-to-Peer Networking and Applications, 8(3), 474-492.

16. Desai, T., \& Prajapati, J. (2013). A survey of various load balancing techniques and challenges in cloud computing. International Journal of Scientific \& Technology Research, 2(11), 158-161

17. Hussain, H., Malik, S. U. R., Hameed, A., Khan, S. U., Bickler, G., Min-Allah, N., Kolodziej, J. (2013). A survey on resource allocation in high performance distributed computing systems. Parallel Computing, 39(11), 709-736

18. Suresh K.C., Haripriya K. and Kruthika S.R."Cooperative Multipath Admission Control Protocol: A Load Balanced Multipath Admission Policy", Research Journal of Biotechnology, Vol. (Special Issue II), August (2017)

19. Suresh K.C, Ashok S, Vishanth.S and Sriram.S "A Machine Learning Approach For Providing Location Aware Services In
Mobile Ad Hoc Networks", International Journal of Pure and Applied Mathematics, Volume 117 No. 21,pp.937-941, 2017 